\title{
Improvement of CH election in three-level heterogeneous WSN
}

\author{
Jong-Yong Lee ${ }^{1}$, Daesung Lee ${ }^{2}$ \\ ${ }^{1}$ Ingenium College of liberal arts, KwangWoon University, Seoul 01897, Korea \\ ${ }^{2}$ Department of Computer Engineering, Catholic University of Pusan, Busan 46252, Korea
}

\begin{tabular}{l}
\hline \hline Article Info \\
\hline Article history: \\
Received Sep 2, 2018 \\
Revised Nov 3, 2018 \\
Accepted Nov 19, 2018 \\
\hline
\end{tabular}

Keywords:

Cluster

Elect

Heterogeneous

Level

Protocol

WSN

\begin{abstract}
A Wireless Sensor Network is a wirelessly configured Sensor Node with limited power such as a battery. There are many Wireless Sensor Network Protocols to increase energy efficiency, among which LEACH Protocol and SEP are typical. The LEACH Protocol is mainly used for homogeneous sensor networks with the same initial energy, and SEP is used for heterogeneous sensor networks with different initial energies. In the case of SEP-E, another heterogeneous sensor with different initial energy is added. SEP and SEP-E provide a higher probability of Cluster Head election for node types with more energy than Normal Nodes. Since the current residual energy of the node is not confirmed, even if the energy is low, the Cluster Head may be elected because of the node type. In this paper, considering the residual energy of a node when a Cluster Head is elected, we increase the probability of electing a Cluster Head with more residual energy. Cluster Head consumes a lot of energy. A node with a lot of residual energy is elected as a Cluster Head, so the network lifetime can be used for a long time.
\end{abstract}

Copyright (C) 2019 Institute of Advanced Engineering and Science. All rights reserved.

\section{Corresponding Author:}

Daesung Lee,

Department of Computer Engineering,

Catholic University of Pusan, Busan 46252, Korea.

Email: dslee@cup.ac.kr

\section{INTRODUCTION}

A Wireless Sensor Network refers to a network in which Sensor Nodes with collection and transmission/reception functions are wirelessly configured. Because the Sensor Nodes are configured wirelessly, each node has a limited energy, such as a battery. Sensor Nodes can be installed in a home, a natural environment, a road, etc. to measure or observe changes in the environment. These networks are used in many areas of everyday life and make our lives more convenient. In addition, since the Sensor Nodes are wirelessly configured, they can be installed where people can not actually go. Wireless Sensor Networks have these advantages, but they also have drawbacks. Unlike a wired network, a Wireless Sensor Network operates with a limited power source such as a battery for each Sensor Node. If the battery is exhausted, the Sensor Node will no longer in function. Therefore, in order to maintain a network for a long time, energy consumption should be minimized by optimizing the energy consumed.

In the Wireless Sensor Network, there are a homogeneous Wireless Sensor Network and a heterogeneous Wireless Sensor Network. In the homogeneous Wireless Sensor Network, all the Sensor Nodes are the same. The heterogeneous Wireless Sensor Network is a mixture of Sensor Nodes having different initial energy. There are many Wireless Sensor Network Protocols to increase the energy efficiency of the network. [1-3] Typical Protocols are LEACH Protocol [4] and SEP [5]. The LEACH Protocol is a cluster-based Protocol that elects a Cluster Head node cyclically using a Cluster Head election formula. SEP is a Protocol for heterogeneous Wireless Sensor Networks based on LEACH Protocol. The SEP distributes the Cluster Heads according to the node type using the different Cluster Head election equations for the Normal Node and Advanced Node, which has more initial energy than the Normal Node. SEP-E [7] added an 
intermediate node with more energy to the SEP, and also gave different election formulas depending on the node type. However, in the case of SEP or SEP-E, the probability of election is increased for nodes with more initial energy considering only the node type without the residual energy. Therefore, the Cluster Head node may be elected a node with low energy. In this paper, we propose a method to solve these problems.

\section{RESEARCH METHOD}

\subsection{Heterogeneous Network}

In a heterogeneous wireless sensor network, the energy of all the nodes is not the same as the homogeneous wireless sensor network where the energy of all the nodes is the same. $m \%$ of all nodes are Advanced Nodes that has more energy than a Normal Node. That is, when the total number of nodes is $n$, $n \times(1-m)$ Normal Nodes having initial energy value $e_{0}$ exist, and there are $n \times m$ Advanced Nodes having more initial energy than Normal Nodes.

\subsection{Three-level Heterogeneous Network}

A three-level heterogeneous wireless sensor network is a network of three types of nodes with different energies. $m \%$ of all nodes are Advanced Nodes with more energy than Normal Nodes and $m_{0} \%$ of the Advanced Nodes are supernodes. In other words, if the total number of nodes is $n$, there are $n \times(1-m)$ Normal Nodes with an initial energy value $e_{0}$ and $n \times\left(1-m_{0}\right)$ Advanced Nodes several $\alpha$ times more than the initial energy. And there are $n \times m \times\left(1-m_{0}\right)$ super nodes that are $\beta$ times as much as the initial energy.

\subsection{LEACH Protocol}

The LEACH Protocol is a Cluster-based routing Protocol. The Sensor Field is divided into Clusters, and there is one Cluster Head node per Cluster. The LEACH Protocol stochastically elects the Cluster Head and gives all the nodes in the Cluster the opportunity to be elected as the Cluster Head. The LEACH Protocol has a set-up phase in which Cluster Head elections are made and a steady-state phase in which transmission is actually effected. The Cluster Head consumes a lot of energy because it collects the data of the member nodes in the Cluster and transmits it to the Base Station. When a node is continuously elected as a Cluster Head, the energy of the node is consumed immediately. Therefore, all nodes can be elected as a Cluster Head by using the threshold equation in the LEACH Protocol. In the set-up phase, the Cluster Head is elected using the following equation.

$$
T(n)=\left\{\begin{array}{cc}
\frac{p}{1-p\left(r \bmod \frac{1}{p}\right)} & \text { if } n \in G \\
0 & \text { otherwise }
\end{array}\right.
$$

As shown in (1), $r$ is the current round. If the set $G$ is empty and there are no more nodes that can become Cluster Heads, put all remaining energy nodes in the set $G$ so that they become Cluster Heads. Set $G$ is the set to which nodes that are not elected to the Cluster Head belong. If the random number is less than the threshold $T(n)$, the node is elected as the Cluster Head in the current round. Once all the Cluster Heads are elected, the member nodes in the Cluster transmit the data to the Cluster Head. The Cluster Head transmits the data of the received member nodes and its own data to the Base Station. Figure 1 shows flowchart of LEACH.

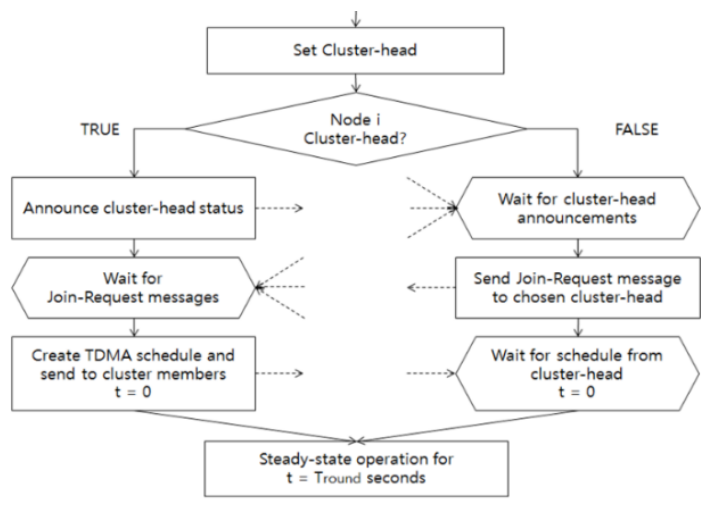

Figure 1. Flowchart of LEACH protocol 


\subsection{SEP}

SEP is suitable for heterogeneous networks and gives a different Cluster Head probability equation depending on the type of nodes. Heterogeneous networks are different from homogeneous networks in which all nodes have the same energy. A node with more energy than a Normal Node is called an Advanced Node.

In case of Cluster Head election, it is elected by probability equation like LEACH Protocol. In case of SEP, weight is applied to Advanced Node with higher energy to increase the election probability. The weighted probability formula of the Normal Node and the weighted probability formula of the Advanced Node are shown in (2).

$$
\begin{aligned}
& p_{\text {nrm }}=\frac{p}{1+\alpha \times m} \\
& p_{a d v}=\frac{p(1+\alpha)}{1+\alpha \times m}
\end{aligned}
$$

The Cluster Head election probability $T\left(s_{n r m}\right)$ and $T\left(s_{a d v}\right)$ using the weighted probability equation of (2) are shown in (3).

$$
\begin{aligned}
& T\left(s_{\text {nrm }}\right)=\left\{\begin{array}{cc}
\frac{p_{\text {nrm }}}{1-p_{\text {nrm }}\left(r \bmod \frac{1}{p_{n r m}}\right)} & \text { if } n \in G \\
0 & \text { otherwise }
\end{array}\right. \\
& T\left(s_{\text {adv }}\right)=\left\{\begin{array}{cc}
\frac{p_{\text {adv }}}{1-p_{a d v}\left(r \bmod \frac{1}{p_{a d v}}\right)} & \text { otherwise } \\
0 & \text { otherwise }
\end{array}\right.
\end{aligned}
$$

As shown in (3), $r$ is the current round and $G$ is the set of Normal Nodes that are not Cluster Head within $\frac{1}{p_{n r m}}$ round of Epoch. And $T\left(s_{n r m}\right)$ is a threshold applied to $n \times(1-m)$ Normal Nodes. This ensures that Normal Nodes are Cluster Heads exactly once every $\frac{1}{p_{\text {opt }}} \times(1+\alpha \times m)$ round of Epoch. The number of average Cluster Heads of the Epoch is $n \times(1-m) \times p_{n r m} . G$ is a set of Advanced Nodes that are not Cluster Heads within the last $\frac{1}{p_{a d v}}$ round of Epoch. And $T\left(s_{a d v}\right)$ is a threshold applied to $n \times m$ Advanced Nodes. This ensures that the Advanced Nodes are Cluster Heads exactly once every $\frac{1}{p_{o p t}} \times \frac{(1+\alpha \times m)}{1+\alpha}$ rounds. This period is defined as a Sub-epoch.

Each Epoch has $1+\alpha$ sub-epoch. As a result, Advanced Nodes in a heterogeneous epoch become Cluster Heads exactly $1+\alpha$ times. The average number of Cluster Heads per round of heterogeneous epoch is $n \times m \times p_{a d v}$.

$$
n \times(1-m) \times p_{n r m}+n \times m \times p_{a d v}=n \times p_{o p t}
$$

As shown in (4), the sum of the average number of Cluster Heads per Normal Node per heterogeneous epoch and the average number of Cluster Heads per Advanced Node per sub-epoch is equal to the average number of Cluster Heads per epoch per round. Figure 2 shows numerical example.

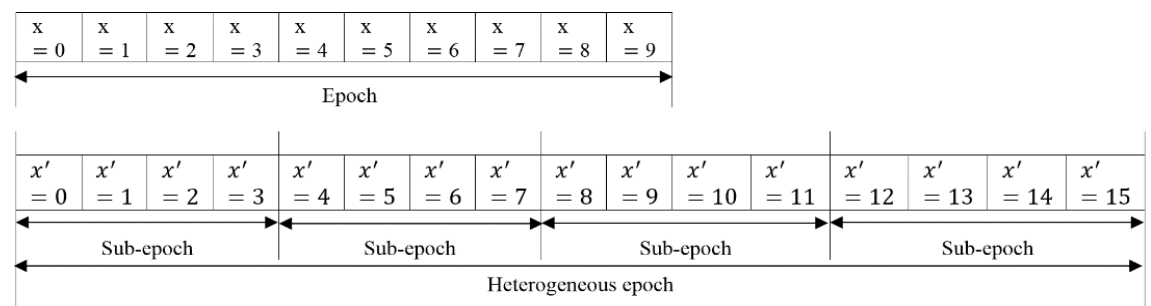

Figure 2. A numerical example for a heterogeneous network

\subsection{SEP-E}

SEP-E is a SEP-based Protocol for three-level heterogeneous sensor networks. Existing SEPs consider only two types of Sensor Nodes with different energy, but SEP-E adds another node type with different energy. It has Normal Node, and Advanced Node that has more energy than Normal Node, intermediate node in which has more energy than Advanced Node. 
Instead of $p$ in (1) of LEACH Protocol, $p_{n r m}$ is assigned to Normal Node, $p_{a d v}$ is assigned to Advanced Node, and $p_{\text {int }}$ is assigned to Intermediate node.

$$
\begin{aligned}
& p_{\text {nrm }}=\frac{p}{1+m\left(\alpha-m_{0}(\alpha-\beta)\right)} \\
& p_{\text {adv }}=\frac{p(1+\alpha)}{1+m\left(\alpha-m_{0}(\alpha-\beta)\right)} \\
& p_{\text {int }}=\frac{p(1+\beta)}{1+m\left(\alpha-m_{0}(\alpha-\beta)\right)}
\end{aligned}
$$

The ratio of Advanced Node and Intermediate node is $m \%$ of the total node. The ratio of intermediate nodes is $m_{0} \%$ of the Advanced Node.

\section{PROPOSED METHOD}

Since the Cluster Head election threshold of the LEACH Protocol does not consider the residual energy of the node, a node with insufficient energy to operate as a Cluster Head node can be elected. To solve these problems, M.J. Handy [6] has improved the Cluster Head election threshold to take into account the residual energy of the nodes. Handy's proposal is shown in (6).

$$
T(n) \times \frac{E_{n_{\text {current }}}}{E_{n_{\text {Max }}}}
$$

As shown in (6) is Threshold considering the residual energy that multiplies the $T(n)$ value of the LEACH Protocol by the residual energy ratio of the node. $T(n)$ has a value between 0 and 1 . In the LEACH Protocol, the random number of each node is compared with the threshold value, and if the value of the threshold equation is greater, it is elected as the Cluster Head. Therefore, if the threshold value exceeds 1 , the Cluster Head is always elected, so the threshold value should not exceed 1 . In (6), $E_{n_{\operatorname{Max}}}$ is the initial energy of the node, and $E_{n_{\text {current }}}$ is the current energy of the node. Therefore, as the residual energy of the node becomes smaller, the value of the threshold equation becomes closer to 0 and it is not elected as the Cluster Head.

In the case of existing heterogeneous sensor network Protocols, the Cluster Head election probability is increased toward the initial energy depending on the node type. Because it has a lot of initial energy, it is suitable as a Cluster Head. However, since the priority of the election probability is determined according to the node type, inefficient clusters are formed when these types of nodes are far from the Base Station. Also, because the residual energy is not considered, the Cluster Head can be elected first because of the node type even the actual residual energy is insufficient. In this paper, we propose an election probability of formula to solve this problem as it follows.

We first consider the residual energy in $T(n)$ of the existing LEACH Protocol. In the case of M.J. Handy, the Cluster Head election probability was adjusted according to the energy ratio by multiplying the election energy by the $\frac{\text { residual energy of the node }}{\text { initial energy of the node }}$.

The smaller the residual energy of a node, the less the Cluster Head election probability. However, if the remaining energy of the nodes is reduced as the network proceeds, the Cluster Head election probability is reduced as a whole, and the Cluster Head may not be elected. To overcome this problem, residual energy ratio of the node is changed based on the maximum residual energy of the alive nodes in the Sensor Field, not based on the initial energy. $\left(\frac{E_{n_{\text {current }}}}{E_{n_{\text {Max }}}}\right.$ to $\left.\frac{E_{n_{\text {current }}}}{\operatorname{MAX}\left(E_{n_{\text {current }}}\right)}\right)$

The proposed Cluster Head election threshold formula applying improvements is shown in (7).

$$
T(n) \times \frac{E_{n_{\text {current }}}}{\operatorname{MAX}\left(E_{n_{\text {current }}}\right)}
$$

\section{SIMULATION AND RESULTS \\ 4.1. Radio Model}

When data is transmitted from the Sensor Node, it requires transmission energy $E_{T X-e l e c}(l)$ and amplification energy $E_{T X-a m p}(l, d)$ depending on the distance. When receiving data from a Sensor Node, it requires receiving energy $E_{R X-e l e c}(l)$. The flowchart of the radio model is shown in Figure 3. 


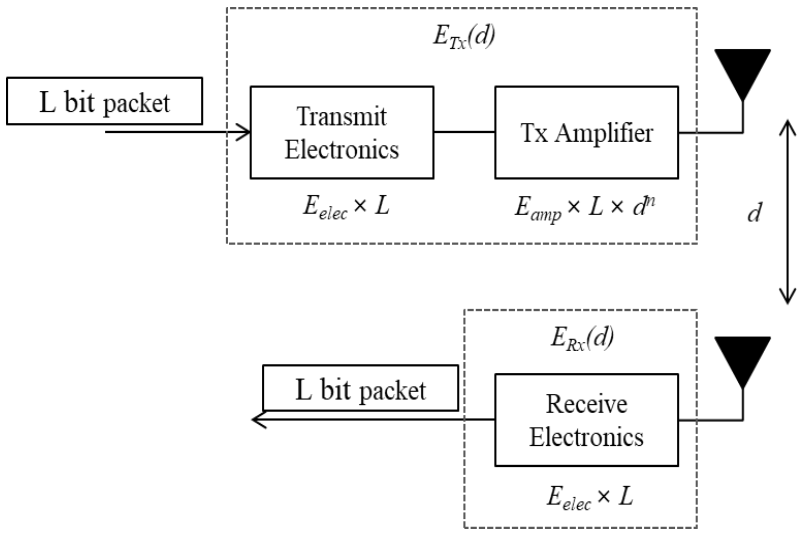

Figure 3. Radio model

Energy consumption is proportional to the square of the distance if the transmission distance is within the free space distance, and energy consumption is proportional to the fourth power of the transmission distance when the transmission distance is outside the free space. The free space range was defined as $d_{0}$. Therefore, in a wireless network, energy consumption increases as the transmission distance increases. This is shown in (8). $l$ is the data packet size, and $d$ is the transmission distance.

$$
\begin{aligned}
& E_{T X}(l, d)=E_{T X-e l e c}(l)+E_{T X-a m p}(l, d) \\
& = \begin{cases}l E_{\text {elec }}+l \varepsilon_{f s} d^{2} & d \leq d_{0} \\
l E_{\text {elec }}+l \varepsilon_{m p} d^{4} & d>d_{0}\end{cases} \\
& d_{0}=\frac{\varepsilon_{f s}}{\varepsilon_{m p}}
\end{aligned}
$$

When receiving data, it requires receiving energy according to the size of the data message. The energy equation required at this time is shown in (9).

$$
E_{R X}(l)=E_{R X-\text { elec }}(l)=l E_{\text {elec }}
$$

The radio model parameters used in the simulation are shown in Table 1.

Table 1. Radio Model Parameters

\begin{tabular}{cc}
\hline Parameters & Value \\
\hline Data Aggregation $\left(E_{D A}\right)$ & $50 \mathrm{~nJ} / \mathrm{bit} / \mathrm{signal}$ \\
Energy dissipation to run the radio device $\left(E_{\text {elec }}\right)$ & $50 \mathrm{~nJ} / \mathrm{bit}$ \\
Free space model of Transmitter Amplifier $\left(\varepsilon_{\mathrm{fs}}\right)$ & $10 \mathrm{pJ} / \mathrm{bit} / \mathrm{m}^{2}$ \\
Multi path model of Transmitter Amplifier $\left(\varepsilon_{m p}\right)$ & $0.0013 \mathrm{pJ} / \mathrm{bit} / \mathrm{m}^{4}$ \\
\hline
\end{tabular}

\subsection{Equal Probability Cluster Head Elected}

Using the unequal probability cluster head election method, on average, the number of cluster heads that are close to the (number of nodes) * (cluster head election probability) is elected. The number of cluster heads is not constant because all the nodes per round elect the cluster head only once according to the critical equation. Occasionally, too many cluster heads may be elected or cluster heads may not be elected at all. This can be no different or less efficient than before clustering. In order to improve this, we use the uniform cluster head election method so that the same number of cluster heads are elected every round. The uniform cluster head selection method elects the cluster head using the critical equation until it becomes (number of nodes) * (cluster head election probability) every round. Figure 4 and Table 2 show the number of cluster heads per round when the total number of nodes is 100 and the number of cluster election probabilities is $10 \%$. 
Table 2. Number of Cluster Heads Per Round

\begin{tabular}{ccc}
\hline Round r & Unequal elect & Equal elect \\
\hline 1 & 16 & 10 \\
2 & 11 & 10 \\
3 & 13 & 10 \\
4 & 11 & 10 \\
5 & 11 & 10 \\
6 & 7 & 10 \\
7 & 11 & 10 \\
8 & 10 & 10 \\
9 & 4 & 10 \\
10 & 6 & 10 \\
Avg. & 10.00 & 10.00 \\
\hline
\end{tabular}
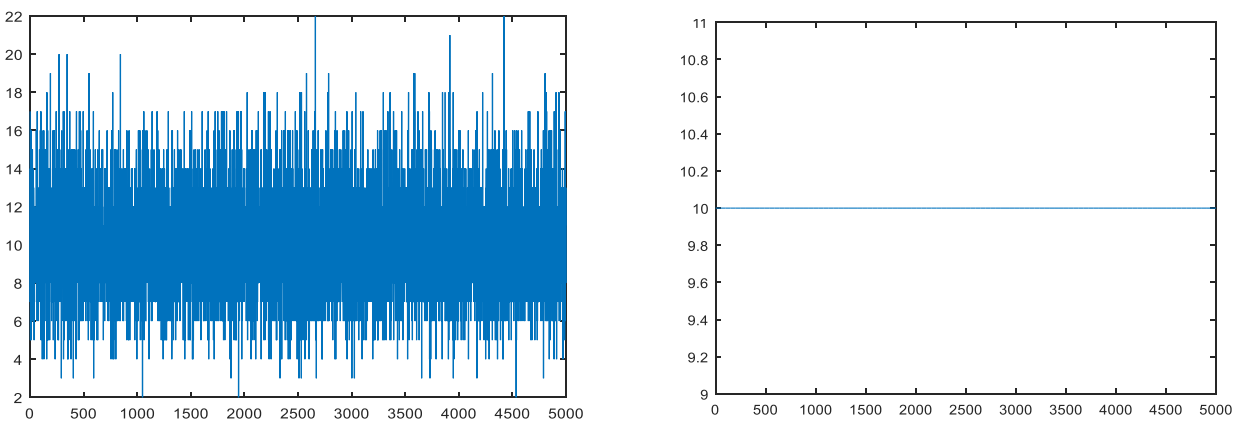

Figure 4. Number of cluster heads per round

\subsection{Simulation}

We compared the network lifetime of the proposed method and the existing LEACH Protocol. The Simulation Parameters are shown in Table 3.

Table 3. Simulation Parameters

\begin{tabular}{cc}
\hline Parameters & Value \\
\hline Number of Sensor Nodes $(N)$ & 100 \\
Sensor Field & $400 \times 400$ \\
Position of Base Station & Center $(200,200)$ \\
Initial Energy $\left(e_{0}\right)$ & $0.5 \mathrm{~J}$ \\
Advanced Node Additional Energy $(\alpha)$ & 1.5 \\
Super Node Additional Energy $(\beta)$ & 3 \\
Ratio of Advanced Node $(m)$ & 0.2 \\
Ratio of Intermediate Node $\left(m_{0}\right)$ & 0.5 \\
Size of Packet & 1000 bits \\
\hline
\end{tabular}

Assuming that the Sensor Field is $400 \times 400$ and the location of the Base Station is located at the center $(200,200)$ of the Sensor Field then the Sensor Nodes are randomly arranged as shown in Figure 5.

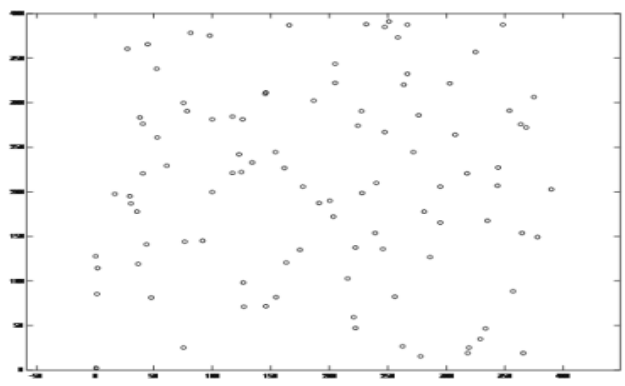

Figure 5. Node placement in sensor field 


\subsection{Simulation Result}

The Figure 6 and Table 4 show the results of network lifetime comparison using the above simulation parameters. Simulation results shows that SEP-E has a 393\% of network lifetime improvement over the LEACH Protocol. When energy is considered in Proposed method, it can be confirmed that it is improved by $22.6 \%$ compared to SEP-E.

Table 4. Simulation Result

\begin{tabular}{ccc}
\hline Protocol & FND & Improved Ratio \\
\hline LEACH Protocol & 45 & \\
SEP-E & 177 & $393 \% \boldsymbol{\Delta}$ than LEACH Protocol \\
Proposed method & $\mathbf{2 1 7}$ & $\mathbf{2 2 . 6 \%} \boldsymbol{\Delta}$ than SEP-E \\
\hline
\end{tabular}

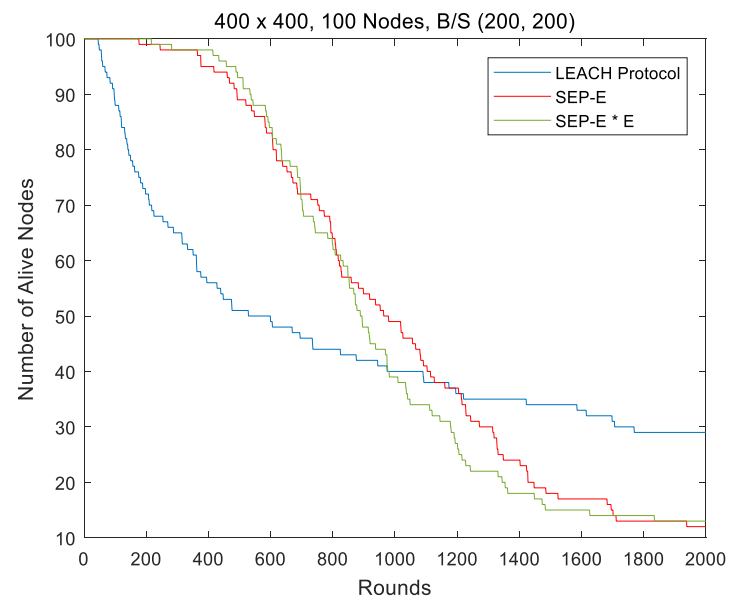

Figure 6. Comparison of network lifetime

\section{CONCLUSION}

Clustering-based Wireless Sensor Network Protocols can help to improve Network lifetime. However, since a Cluster Head of the cluster aggregates and transmits data, the energy burden is great. Heterogeneous sensor network is composed of nodes that have different initial energy. In order to maintain the Network lifetime of the network for a long time, it is effective to elect nodes having high initial energy as Cluster Head.

However, if the Cluster Head election probability is increased only for nodes with a lot of initial energy, a node with a low energy may be elected as a Cluster Head, which may be inefficient. In this paper, we consider the residual energy to solve this problem, also the improvement of the actual Network lifetime.

\section{REFERENCES}

[1] I.F. Akyildiz, W. Su, Y. Sankarasubramaniam, E. Cayirci. A Survey on Sensor Networks, IEEE Communications Magazine. 2002.

[2] J.Y. Lee, K. Jung, H. Jung, D. Lee. Improving the Energy Efficiency of a Cluster Head Election for Wireless Sensor Networks, International Journal of Distributed Sensor Networks. 2014; 10(3).

[3] J.Y. Lee, K. Jung, B. Shrestha, J. LEE, S. Cho. Energy Efficiency Improvement of the of a Cluster Head Selection for Wireless Sensor Networks, International Journal of Smart Home. 2014; 8(3); 9-18.

[4] W. Heinzelman, A. Chandrakasan, H. Balakrishnan. Energy-Efficient Communication Protocol for Wireless Microsensor Networks, Proceedings of the 33rd Hawaii International Conference on System Sciences. 2000.

[5] G. Smaragdakis, I. Matta, A. Bestavros. SEP: A Stable Election Protocol for clustered heterogeneous Wireless Sensor Networks, Boston University Computer Science Department. 2004.

[6] M.J. Handy, M. Haase, and D. Timmermann. Low energy adaptive clustering hierarchy with deterministic clusterhead selection, Mobile and Wireless Communications Network, 2002. 4th International Workshop on. IEEE, 2002.

[7] F.A. Aderohunmu, J.D. Deng, "An Enhanced Stable Election Protocol (SEP) for Clustered Heterogeneous WSN"

[8] W.S. LEE, K.D. Jung, J.Y. Lee. Improvement of Cluster Head selection of LEACH Protocol, International Journal of Applied Engineering Research (IJAER). 2017; 12(20). 